\title{
Hilar fino: Reflexiones, debates y dilemas metodológicos en investigaciones feministas PRESENTACIÓN
}

\author{
ItZiar Gandarias GoikoetXea \\ Universidad de Deusto \\ itziar.gandarias@deusto.es (ESPAÑA) \\ Karina Fulladosa Leal \\ Universidad de la República- Uruguay \\ karinafulladosa@gmail.com (URUGUAY) \\ Daniela Osorio Cabrera \\ Universidad de la República- Uruguay \\ dosorio@psico.edu.uy (URUGUAY)
}

En las últimas décadas, las críticas feministas a la ciencia positivista han agudizado el debate sobre las formas de producción de conocimiento al cuestionar su pretensión de generalización y universalismo, así como la distancia y desafección respecto a lo que tradicionalmente se ha llamado "objetos de estudio". Bajo el paradigma del actual modelo neoliberal, la producción de conocimiento se ve permeada por las lógicas productivistas, sexistas y coloniales en sus formas de hacer, atravesando espacios académicos y activistas de producción. Sin embargo, este paradigma ha sido cuestionado desde el movimiento feminista en su amplitud, recordándonos otros devenires tanto ontológicos como metodológicos, posibilitando y re-construyendo desde la epistemología y su relación con la ciencia, otras difracciones (Haraway, 1995).

Las epistemologías feministas en su pluralidad, no sólo señalan el sesgo de género en la ciencia (Keller y Longino, 1996) y enfocan la mirada desde posiciones invisibles situadas en los márgenes (Harding, 1996), sino que hacen una apuesta contundente por la multiplicidad de producciones locales partiendo de reflexiones en torno a la política de la localización (Rich, 2003) y los conocimientos situados y parciales (Haraway, 1995). La propuesta es una ciencia que aboga por hacer visible el lugar desde el que se mira y las relaciones de poder que se entrecruzan en cualquier forma de conocimiento. 
Sin embargo, el proyecto feminista de crítica metodológica y epistemológica no es lineal ni está cerrado, sino más bien sigue inacabado con debates aún en discusión. Hablamos por ejemplo de los dilemas éticos y metodológicos que surgen en torno a la gestión de las dinámicas de poder en los procesos de investigación o las estrategias para fortalecer el impacto de la investigación feminista dentro y fuera de la academia. Efectivamente, las epistemologías feministas, conformadas por perspectivas plurales e incluso en algunos aspectos confrontadas, albergan un corpus de conocimiento y de reflexiones muy rico, abundante y cada vez más transdisciplinar. No obstante, mayoritariamente se trata de aportaciones de corte teórico y apenas disponemos de herramientas prácticas que sirvan de referencia y orientación a la hora de llevar a cabo investigaciones feministas.

Como señalan en la introducción del monográfico "Experiencias de investigación feminista: propuestas y reflexiones metodológicas" (Martínez et al 2014), entrar en la llamada "cocina de las investigaciones" y hacer transparentes las decisiones analíticas y metodológicas es todavía una práctica poco habitual. Por ello, consideramos que, destapar "lo que nos ocurre" durante los procesos de investigación y dar cuenta de las dudas, dilemas e incomodidades que nos atraviesan, es una oportunidad de aprendizaje y de revisión crítica fundamental para fortalecer y mejorar la calidad de las investigaciones que realizamos.

Esta publicación quiere ser una contribución en este sentido. Las autoras de este monográfico nos abren las puertas de "la cocina" de sus investigaciones para compartir sus reflexiones epistemológicas y dilemas metodológicos surgidos durante el proceso. La propuesta es hilar fino para profundizar en temas por los que solemos pasar de puntillas, como, por ejemplo: de qué manera transitar las tensiones que surgen en investigaciones activistas feministas, qué lugar otorgar al cierre de las investigaciones con las participantes, qué espacios hay para la emergencia de nuevas metodologías, qué lugar ocupan los afectos en la investigación y cómo dar cuenta de ellos o cómo transformar la política del reconocimiento en las investigaciones activistas.

Las autoras que participamos en este monográfico compartimos el interés en la producción de un conocimiento comprometido con la transformación social. Esto conlleva que algunas nos movamos en los espacios fronterizos entre academia y militancia; con todas las potencialidades, pero también con todas las tensiones e incomodidades que ello supone. En ese sentido, reivindicamos una posición híbrida y movediza para desde ahí establecer puentes y generar articulaciones entre dos mundos que apenas se miran.

La conformación del propio monográfico también ha implicado un reto encarnado de producir conocimiento en el equilibrio entre subsistir en la academia y la precariedad de la existencia. Nuestras vidas han sido atravesadas por una pandemia, donde ha emergido, con mayor crudeza y visibilidad las crisis que ya hace tiempo los feminismos venimos denunciando, en particular la crisis ecológica, de cuidados y reproducción del sur global (Pérez-Orozco, 2015). A su vez, en el recorrido de estos largos meses hemos sido testigo de nuestras propias etapas vitales y sus retos. Dos de nosotras hemos sido atravesadas por la experien- 
cia de la maternidad, lo que ha supuesto sostener las ambivalencias de la intensa, pero a la vez compleja tarea de maternar mientras nos acompañábamos en la coordinación de este proceso de publicación. Se suman nuestros propios trabajos remunerados transformados y enrarecidos por las actuales circunstancias, donde las fronteras con el descanso, el ocio y los cuidados son cada vez más difusas y la corresponsabilidad sigue siendo una necesidad imperiosa. La distancia desde donde estamos tejiendo este monográfico con las respectivas diferencias horarias entre el Norte y el Sur global, nos ha supuesto otro reto, intentando generar dinámicas de trabajo y reuniones sostenibles para todas. Cada una de estas circunstancias también ha formado parte de este proceso y su análisis sigue alimentando las conversaciones, enfrentando nuestros miedos, contradicciones y posibilidades en la producción de conocimiento colectivo.

Estas diversas experiencias sumadas a los aportes de los artículos que se presentan, vuelven a cuestionar la dimensión emocional y afectiva que atraviesa e imbrica todo proceso de investigación. Visibilizar y nombrar esta dimensión es fundamental para otorgarle valor y legitimidad epistémica en los procesos de investigación feminista. Por ello, este monográfico es también un ejercicio de reflexividad colectiva sobre los afectos y las emociones en los procesos de investigación, ya que en determinadas circunstancias, ocultar estos aspectos, puede estar invisibilizando todo un bagaje y fuente de experiencias y conocimiento que por un lado, nos proporciona incomodidades y tensiones pero, por otro lado, nos habilita a gestar otros diálogos y transformaciones posibles. Proponemos, entonces, una alianza articulatoria entre diferentes posiciones y subjetividades afectivas dignas de ser compartidas en la producción de conocimiento.

El presente número monográfico está compuesto de seis artículos, estructurados en dos partes. Una primera parte, aborda reflexiones teóricas a partir de las epistemologías feministas colocando algunos de los debates centrales actuales cómo el valor epistémico de las emociones, o las implicaciones éticas, políticas y metodológicas de las investigaciones feministas activistas. En una segunda parte, a partir de experiencias concretas de investigación se presentan los dilemas surgidos y las estrategias y acciones llevadas a cabo. Las autoras nos enseñan la trastienda de sus investigaciones y abren caminos necesarios para pensar el abordaje metodológico feminista. El cierre del monográfico incluye un artículo que sistematiza los principales desafíos metodológicos que enfrentan las investigaciones feministas y plantea una guía de orientaciones para futuras investigadoras.

En el primer artículo "Un viaje por las emociones en procesos de investigación feminista" Dau García Dauder y Maris G. Ruiz Trejo reflexionan sobre la relación inseparable entre conocimiento y emoción. Partiendo del concepto de reflexividad fuerte de las epistemologías feministas las autoras enfatizan el valor epistémico de las emociones en los procesos de investigación. Para ello, nos invitan a un viaje reflexivo sobre cómo las emociones de quienes investigan afectan al proceso de investigación, pero también a repensar como el propio proceso afecta emocionalmente a las investigadoras. A través de un diálogo con diferentes autoras exponen las dificultades que existen hoy para discutir sobre emociones en los procesos de investigación además de señalar los inconvenien- 
tes de enfatizar las emociones. El artículo acaba con la necesidad de crear una cultura de reflexividad colectiva donde compartir con otras los dilemas éticos y el trabajo emocional que implica la investigación y así, utilizar las emociones como conocimiento para la transformación social.

En el segundo artículo Daniela Osorio Cabrera, Itziar Gandarias Goikoetxea y Karina Fulladosa Leal nos proponen un recorrido por las: "Consideraciones ético-político-afectivas en investigaciones feministas: articulaciones situadas entre academia y activismo". Este artículo profundiza en el entramado de las epistemologías feministas para dar cuenta de otras formas de hacer e incluso de la posibilidad de crear nuevas metodologías y formas de acercarnos al conocimiento en los procesos de investigación-activista. Para ello, desgranan tres de las dimensiones claves para pensar las investigaciones feministas desde lo ético, lo político y lo afectivo colocando sus experiencias de investigación como una fuente más de conocimiento. A su vez, recuperan la dimensión afectiva como parte transversal de dicho proceso, enunciando las implicaciones que devienen en dicha apuesta política entre los diferentes sujetos que la conforman. Para cerrar, proponen la Amistad como una herramienta ético-política tanto del acompañamiento como de la articulación de un diálogo inacabado entre diferentes para la producción de conocimiento.

En el tercer artículo, "Implicaciones teórico, políticas y metodológicas de la investigación activista feminista", Ana María Castro Sánchez suma también una reflexión de las implicaciones teóricas, políticas y metodológicas de la investigación activista feminista. Toma como referencia de articulación su propio proceso de investigación, basado en las producciones narrativas como propuesta metodológica desde los conocimientos situados. La autora nos propone una reflexión teórica articulada, desplegando los planteos centrales de las epistemologías feministas en conexión con las investigaciones feministas que se caracterizan por el compromiso activista. De este último, destaca las posibilidades, límites $\mathrm{y}$ tensiones que implica una estrategia activista feminista. Como cierre de su propuesta, la autora nos invita a fortalecer los procesos de reflexividad en estas investigaciones, a construir una mirada que más que reflejar difracte, y más que distanciar conecte y articule, comprometiendo nuestro cuerpo-pensamiento en el proceso.

El cuarto artículo escrito por Flor Carina Vargas Martínez y Alejandra Araiza Díaz titulado: "Acción política frente a la violencia feminicida en México. Experiencias de una Investigación Activista Feminista" nos acerca a la trastienda de las investigaciones feministas. Así parten por las reflexiones acerca de las dudas, estrategias y desafíos que han podido identificar en su proceso de investigación a partir de un abordaje desde las epistemologías feministas y de la investigación-activista. Las autoras comienzan compartiendo sus propios recorridos y disyuntivas acerca de la violencia feminicida, siguiendo con las decisiones y transformaciones que en el transcurso de la investigación tuvieron que realizar para llegar a imaginar otras maneras de vincularse con el conocimiento. Desde este lugar nos acercan a las experiencias, saberes y resistencias de las activistas, colectivos y familias que se articulan en relación a dicha temática. El artículo 
concluye con algunas consideraciones que nos permiten vislumbrar y compartir algunas estrategias y dilemas de la Investigación Activista Feminista para que puedan ser de utilidad e inspiración para futuras investigadoras y creación de políticas públicas al respecto.

El quinto artículo de Estibaliz de Miguel Calvo, "Se Busca". Métodos de investigación para una aproximación feminista a mujeres detenidas por la policía", se pregunta por la posibilidad de poner en práctica métodos feministas para analizar la experiencia de las mujeres detenidas en calabozos en la Comunidad Autónoma Vasca. Preocupada por el impacto personal y emocional que tiene relatar en primera persona la experiencia de detención policial y los pasos a seguir para acompañar el proceso, analiza los métodos desde una perspectiva ética coherente con los planteos feministas en la investigación. Describe los debates, las dudas y las decisiones tomadas por el equipo de investigación para seleccionar la estrategia de abordaje. Destaca en la elección, la estrategia del grupo de cierre como posibilidad para construir un espacio "amable" que permite a las participantes de la investigación, compartir las vivencias, emociones, temores vinculados a la experiencia de criminalización y paso por el sistema. La estrategia, demuestra la centralidad de la interacción en la producción de conocimiento, y el abordaje de las emociones como parte sustancial de los procesos de investigación.

Por último, Rocío Jimenez Cortés cierra el monográfico con el artículo "Diseño y desafíos metodológicos de la investigación feminista en ciencias sociales". En él, se propone en primer lugar, generar una guía metodológica para el diseño de investigaciones feministas en las diferentes áreas de Ciencias Sociales que pueda servir como material didáctico para estudiantes. En segundo lugar, revisa en la literatura científica actual los principales desafíos metodológicos que enfrenta hoy la investigación feminista identificando cuatro retos: a) la incorporación de la interseccionalidad, b) la inclusión de la reflexividad como criterio de rigor, c) la generación de prácticas éticas en torno a la agencia de las participantes y d) el papel de las emociones en las investigaciones feministas. En definitiva, el artículo tiene como finalidad abrir líneas de debate actuales en la comunidad científica que contribuyan tanto a la puesta en práctica de investigaciones feministas como a la docencia.

El tejido que compone este monográfico, abre las puertas de nuestros recorridos para compartirse, de manera generosa como intercambio con quienes se acerquen a estos textos. Decimos tejido como metáfora que conecta nuestras posiciones en el proceso de investigación, señalando los nudos, las costuras y los remiendos que componen la tarea de investigar como feministas. Esperamos que, para ustedes, como para nosotras sea la posibilidad de seguir hilando juntas.

\section{BIBLIOGRAFÍA}

HARAWAY, DONNA (1995): Ciencia, Cyborgs y Mujeres. La Reinvención de la Naturaleza, Madrid, Cátedra. 
KELLER, EVELYN FOX AND LONGINO, HELEN E. (1996). Feminism and Science, Oxford: University Press,

HARDING, SANDRA (1996): Ciencia y Feminismo, Madrid, Morata.

MARTÍNEZ MARTÍNEZ, LUZMA., BIGLIA, BARBARA., LUXÁN, MARTA., FERNANDEZ, CRISTINA., ASPIAZU, JOKIN \& BONET Y MARTÍ, JORDI. (2014). Experiencias de investigación feminista: propuestas y reflexiones metodológicas. Athenea Digital: Revista de pensamiento e investigación social, 14(4), pp. 3-16.

PÉREZ-OROZCO, AMAIA (2014): Subversión feminista de la economía, Madrid,Traficantes de Sueños.

RICH, ADRIENNE. (2003): Notes towards a politics of location. Feminist postcolonial theory: A reader, pp.29-42. 